\title{
Análise espacial da umidade do solo cultivado com soja sob dois sistemas de manejo ${ }^{1}$
}

Syonei V. Zanette' ${ }^{2}$, Silvio C. Sampaio ${ }^{2}$, Marciane G. Silvestre², Márcio A. V. Boas², Miguel A. Uribe-Opazo² \& Manoel M. F. de Queiroz ${ }^{2}$

\section{RESUMO}

Abordaram-se, neste trabalho, aspectos básicos da geoestatística para análise espacial de dados de umidade do solo, com ênfase na análise de semivariograma como metodologia para determinação da dependência espacial. O objetivo consistiu na análise da umidade sob condições de campo, na região de Toledo, PR, em solo argiloso, nas profundidades de 15 e $30 \mathrm{~cm}$, sob sistema de manejo plantio direto e preparo convencional do solo; o local da pesquisa foi constituído de duas áreas de $14 \times 14$ m. Realizou-se, em cada área do experimento, um levantamento por amostragem em uma malha de 64 pontos espaçados $2 \mathrm{~m}$, e se utilizou, para análise das amostras, o método convencional (gravimétrico). Os dados foram analisados através da estatística clássica e da geoestatística. Os resultados indicaram baixo coeficiente de variação em todas as coletas de dados, o que indica homogeneidade dos dados. A análise dos semivariogramas indicou dependência espacial da umidade entre moderada e fraca. Os alcances da dependência espacial foram de 5,20 a 10,67 m para a umidade do solo. Os valores de umidade estão relacionados com a profundidade em ambos os sistemas de manejo mas as variabilidades não acompanharam esta tendência.

Palavras-chave: semivariograma, geoestatística, dependência espacial

\section{Spatial analysis of soil moisture with soybean under two management systems}

\begin{abstract}
In this work basic aspects of geoestatístics were approached for spatial analysis of soil moisture data, with an emphasis on semivariogram analysis as a methodology for determination of spatial dependence. The objective consisted of moisture analysis under field conditions in Toledo, Paraná State, Brazil, in clayey soils, in depths of 15 and $30 \mathrm{~cm}$, under management systems of no tillage and conventional planting. The research site constituted of two areas of $14 \times 14 \mathrm{~m}$. In each area of the experiment, sampling in a mesh of 64 spaced points of $2 \mathrm{~m}$ took place. The data were used for analysis of moisture using the conventional gravimetric method. The data were analyzed through the classic statistics and geostatistics. The results indicated a low variation coefficient in all data collections, which indicates homogeneity of the data. The analysis of the semivariograms showed spatial dependence of the moisture variable as being moderate to weak. The reaches of spatial dependence were from 5.20 to $10.67 \mathrm{~m}$ for the soil moisture. For this variable, the values are related with depth in both management systems, but the variabilities did not show this tendency.
\end{abstract}

Key words: semivariogram, geoestatístics, spatial dependence

1 Parte da Dissertação de Mestrado do primeiro autor apresentada ao Programa de Pós-Graduação em Engenharia Agrícola/UNIOESTE

2 RHESA/UNIOESTE, Rua Universitária, 2069, CEP 85819-110, Cascavel, PR. Fone: (45) 3220-3262. E-mail: syonei@unipar.br; ssampaio@unioeste.br; marci.sil@ibest.com.br; vilasma@unioeste.br; mopazo@unioeste.br; mfqueiroz@unioeste.br 


\section{INTRODUÇÃO}

A alimentação da humanidade depende, quase que totalmente, do cultivo do solo arável, uma camada de 10 a $20 \mathrm{~cm}$ de profundidade, denominada camada fértil; sua destruição pode ser conseguida em curto intervalo de tempo se os métodos de manejo não forem corretos para aquele tipo de solo e culturas a serem implantadas. Decorre, daí, a necessidade de se conservar produtiva a camada arável do terreno; é conveniente, então conservar a água, oferecer possibilidade de circulação do ar e manter a fertilidade.

No Brasil, o plantio direto vem sendo, há alguns anos, uma alternativa de conservação dos solos, proporcionando economia nos custos de produção e diminuindo o tempo no preparo e semeadura mas algumas características do solo ainda não definiram em qual sistema de manejo se apresentam ideais, contribuindo para o melhor ciclo e produtividade da cultura. Dependendo das condições de uso e manejo, as características físicas do solo podem modificar-se, evoluindo para situações positivas ou negativas ao crescimento das plantas e produtividade.

As propriedades físicas do solo são interdependentes, a ocorrência de modificações em uma delas leva, normalmente, à mudança em todas as outras; assim, mudanças na estrutura do solo pelo preparo acarretam mudanças também na porosidade, tamanho de poros, retenção e armazenamento de água, entre outras.

Entre os componentes de manejo, o preparo do solo pode ser a atividade que mais influi no comportamento físico, uma vez que atua diretamente sobre a estrutura e, sendo esta uma propriedade que interage ou afeta uma série de outras características do perfil, sua modificação altera praticamente todas as variáveis a ela ligadas.

A estatística clássica de dados supõe que as variáveis aleatórias são independentes entre si, ou seja, as observações vizinhas não exercem influências umas sobre as outras; o que poderá ser feito é a verificação da dispersão dos dados, possibilitando analisar a dispersão dos valores observados em relação à média. $\mathrm{O}$ comportamento de uma variável apresenta-se com estruturação nas variações entre vizinhos, o que torna essas variações independentes e, portanto, apresentam algum grau de dependência espacial (Cressie, 1991).

O estudo da variabilidade espacial e temporal de uma variável de interesse pode ser feito através de técnica denominada geoestatística a qual, a partir de alguns pressupostos, analisa as correlações entre as observações (Issaks \& Srivastava, 1989). A caracterização da variabilidade espacial das propriedades físicas do solo, associada a outras técnicas estatísticas, pode contribuir para a tomada de decisões sobre a implantação de uma cultura que forneça maior rentabilidade e proporcione a conservação dos solos (Vieira, 1998).

O presente trabalho foi realizado com o objetivo de avaliar a variabilidade espacial da umidade da água no solo, em duas profundidades, sob dois sistemas de plantio, direto e convencional, durante o ciclo da soja, na região de Toledo, PR, no ano de 2001/2002.

\section{MATERIAL E MÉTODOS}

Os solos da região onde foi instalado o experimento estão diretamente condicionados à sua geologia e geomorfologia; neste caso, a toposseqüência predominante nas áreas de relevo plano e suave ondulado, se compõe de solos profundos, com baixa susceptibilidade à erosão, bem drenados nos terços superior e médio e imperfeitamente drenados a mal drenados, no terço inferior. Nas áreas de relevo forte ondulado a montanhoso, ocorrem solos férteis, porém rasos e pedregosos, com severas restrições para uso agrícola (EMBRAPA, 2000).

A área experimental está localizada na Linha Cerâmica Prata, município de Toledo, PR, onde predomina o Latossolo Vermelho Distroférrico profundo, com textura argilosa e capacidade de armazenamento de água entre 0,8 e $1,2 \mathrm{~mm} \mathrm{~cm}^{-1}$ de profundidade (EMBRAPA, 2000).

A área de pesquisa foi constituída de dois talhões (cada um com malha de 64 pontos $(8 \times 8)$, espaçados $2 \mathrm{~m}$ ) de $14 \times 14 \mathrm{~m}$, totalizando $196 \mathrm{~m}^{2}$, talhões com declividades médias de 3\%; um talhão recebeu plantio direto e o outro preparo convencional do solo, ambos cultivados com soja, no período de verão. $\mathrm{O}$ espaçamento de $2 \mathrm{~m}$ entre os pontos amostrais e o número de 64 amostras, foram adotados para se garantir a existência de estacionaridade intrínseca e gerar um número mínimo de 30 pares para cada distância em estudo, para utilizar a geoestatística (Clark, 1979; Cressie, 1991).

O preparo convencional do solo foi limitado em escarificação entre 40 e $50 \mathrm{~cm}$ de profundidade, gradagem profunda e semeadura, enquanto o plantio direto foi feito sobre a rebrota da aveia, sendo o primeiro ano de plantio direto no local. Os tratos culturais, controle de ervas e controle de insetos, foram concomitantes para os dois sistemas.

Os dados de precipitação foram coletados em pluviômetro instalado na área e confrontados com os dados da Secretaria do Meio Ambiente e Recursos Hídricos do Estado do Paraná (Figura 1).

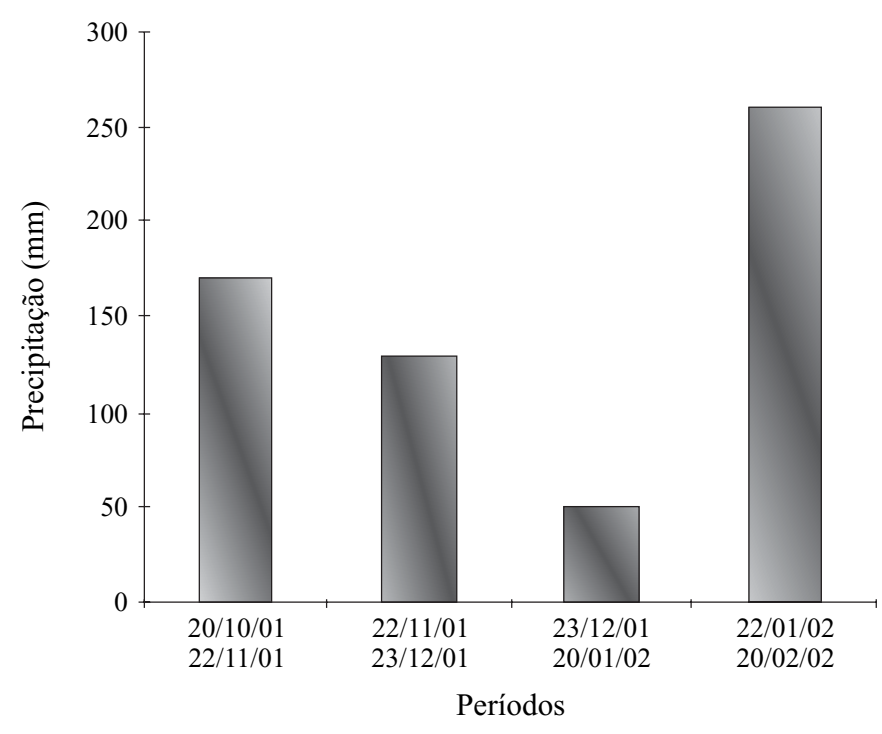

Figura 1. Precipitações na área experimental durante o ciclo da cultura 
Coletaram-se quatro amostras de solo para determinação de umidade durante o ciclo da cultura em ambos os talhões espaçadas, em média, 30 dias, com a primeira coleta 32 dias após o plantio. As coletas foram realizadas durante o ciclo da cultura, nas profundidades de 15 e $30 \mathrm{~cm}$, com 64 dados para cada profundidade, distanciados $2 \mathrm{~m}$. O método usado para análise das amostras foi o convencional (gravimétrico), o qual exige coletas mecânicas, fazendo-se uso de trado e células de acondicionamento do material, pesagem e secagem em estufa a $105 \pm 3{ }^{\circ} \mathrm{C}$ por $24 \mathrm{~h}$, conforme EMBRAPA (1997).

Realizaram-se as análises exploratórias de dados visando caracterizar a distribuição de probabilidade, verificar a variabilidade dos dados de umidade da água no solo e comparar os sistemas de manejo em plantio direto com o sistema convencional. As estatísticas utilizadas nesta análise, foram: média $(\overline{\mathrm{X}})$, desvio padrão $(\mathrm{S})$, coeficiente de variação $(\mathrm{CV})$, coeficiente de assimetria (Cs) e coeficiente de curtose (Cc). As medidas de dispersão proporcionaram visualizar a dimensão da dispersão dos valores observados em relação à média. Os valores mínimos, máximos e amplitude dos dados, também foram analisados para cada variável proporcionando a visualização da maior e menor ocorrência. A assimetria e curtose foram utilizadas para estudar a forma da distribuição de probabilidade das variáveis em estudo. Os coeficientes de assimetria e curtose amostrais foram comparados com os intervalos de confiança da assimetria e curtose gerados para a diferentes tamanhos de amostras nos casos de haver distribuição normal de probabilidade (Jones, 1969). A normalidade das variáveis também foi verificada pelo teste estatístico de Anderdon-Darling.

Em relação às variáveis que não apresentaram distribuição normal, procedeu-se à transformação Box \& Cox (1964) dos dados (Eq. 1).

$$
\mathrm{Z}^{*}(\mathrm{x})=\mathrm{h}_{\lambda}(\mathrm{z})=\left[\begin{array}{ll}
\frac{\mathrm{Z}^{\lambda}(\mathrm{x})-1}{\lambda} & \text { se } \lambda \neq 0 \\
\log (\mathrm{z}(\mathrm{x})) & \text { se } \lambda=0
\end{array}\right.
$$

em que:

$$
Z^{*}(x) \text { - é a variável transformada }
$$

$\lambda$ - é um parâmetro de transformação desconhecido, sendo $-2 \leq \lambda \leq 2$ (a determinar)

A geoestatística foi utilizada com o objetivo de definir a existência ou não da estrutura de variabilidade espacial da umidade do solo.

As características e as propriedades dos solos possuem variabilidades que podem ser casuais, não se sabendo se existe explicação para sua ocorrência, ou variações que podem ser explicadas pelas tendências direcionais manifestadas pelos processos de formação do solo e pelos tipos de uso e manejo dos mesmos.

A análise da variabilidade espacial neste trabalho foi realizada a partir da análise geoestatística do semivariograma, que permite estudar a autocorrelação entre pontos amostrais de uma variável regionalizada, considerando-se sua posição espacial.

O semivariograma é a representação gráfica da função semivariância experimental versus distância entre pontos (h).
A distância máxima recomendada para construir o semivariograma, é de até $50 \%$ da distância máxima da área em estudo (Clark, 1979).

Os valores de semivariâncias experimentais são obtidos pela equação:

$$
\gamma(h)=\frac{\sum\left[Z\left(s_{i}\right)-Z\left(s_{i}+h\right)\right]^{2}}{2 N(h)}
$$

em que:

$\gamma($ h) - é a semivariância experimental, obtida pelos valores amostrados

h - distância entre os pontos amostrais

$\mathrm{N}(\mathrm{h})$ - número total de pares possíveis, dentro da malha de amostragem, com a distância $h$; e $\mathrm{s} \in \mathrm{S} \subset \mathrm{R}^{2}$

Para uso do método geoestatístico, deve-se verificar a suposição de estacionaridade intrínseca da variável em estudo. A variável $\mathrm{Z}(\mathrm{s})$ refere-se às ocorrências da variável $\mathrm{Z}$, com referenciação no espaço paramétrico $(\mathrm{S})$. O método para se ajustar um modelo teórico ao semivariograma experiental usado neste trabalho, foi o "Indicative of Goodness of Fit" - IGF, sugerido por Pannatier (1996), calculado pela soma dos quadrados dos resíduos ponderados, conforme a Eq. 3.

$$
\mathrm{IGF}=\frac{1}{\mathrm{~N}} \sum_{\mathrm{K}=1}^{\mathrm{n}} \sum_{\mathrm{i}=0}^{\mathrm{n}(\mathrm{K})} \frac{\mathrm{P}(\mathrm{i})}{\sum_{\mathrm{j}=0}^{\mathrm{n}(\mathrm{K})} \mathrm{P}(\mathrm{j})} \times \frac{\mathrm{D}(\mathrm{K})}{\mathrm{d}(\mathrm{i})} \times\left[\frac{\gamma(\mathrm{i})-\hat{\gamma}(\mathrm{i})}{\sigma^{2}}\right]^{2}
$$

em que:

$\mathrm{N}$ - número de semivariogramas direcionais

$\mathrm{n}(\mathrm{K})$ - número de lags relativo ao k-ésimo semivariograma

$\mathrm{D}(\mathrm{k})$ - distância máxima no k-ésimo semivariograma

$\mathrm{P}(\mathrm{i})$ - número de pares do i-ésimo lags do k-ésimo semivariograma

d(i) - distância média dos pares do i-ésimo lags do k-ésimo semivariograma

$\gamma(\mathrm{i})$ - valor real da semivariância para o i-ésimo lags

$\hat{\gamma}(\mathrm{i})$ - valor experimental da semivariância para o i-ésimo lags

$\sigma^{2} \quad$ - variância dos dados

Sendo o lags a distância previamente definida e utilizada no cálculo das semivariâncias.

O grau de dependência espacial foi avaliado através da razão entre o efeito pepita $\left(\mathrm{C}_{0}\right)$ e o patamar $\left(\mathrm{C}_{0}+\mathrm{C}_{1}\right)$ (Souza et al., 1999). Os cálculos das semivariâncias para escolha do modelo de semivariograma e os ajustes dos semivariogramas, foram feitos pelo programa computacional de geoestatística Variowin 2.2 (Pannatier, 1996). Elaboraram-se os mapas temáticos da variável umidade do solo para verificar o comportamento espacial dessa variável nos dois sistemas de manejo e nas duas profundidades; foram confeccionados usando-se a interpolação por krigagem ordinário (Krige, 1951), interpolando valores das variáveis em estudo nas posições não amostradas utilizou-se, para a construção desses mapas, o software Surfer 6.0 (Golden Software, 1997). O propósito da krigagem é estimar valores para 
locais não amostrados considerando-se a densidade dos pontos por unidade de área, distância entre os pontos amostrais e variância mínima dos dados, possibilitando a construção de mapas de contorno com alta precisão, além de oferecer limites de variações para os mapas temáticos.

\section{RESULTADOS E DISCUSSÃO}

Apresenta-se, na Tabela 1, a umidade média do solo, a qual teve amplitudes mínimas e máximas entre 28,41 e $31,34 \%$ para o plantio direto (PD) na profundidade de $15 \mathrm{~cm}$, 27,56 a $31,34 \%$ para o preparo convencional (PC) na profundidade de $15 \mathrm{~cm}, 28,94$ a 33,91\% para plantio direto a $30 \mathrm{~cm}$ e 27,41 a $34,02 \%$ no preparo convencional $30 \mathrm{~cm}$. Observa-se maior amplitude de umidade na profundidade de $30 \mathrm{~cm}$ em relação a $15 \mathrm{~cm}$, em ambos os sistemas. O coeficiente de variação apresentou variabilidade aproximadamente constante em todas as amostragens, inferior a $11 \%$.

Tabela 1. Estatística dos dados de umidade de água no solo referentes aos sistemas de manejo em plantio direto (PD) e preparo convencional (PC) em quatro épocas do cultivo da soja

\begin{tabular}{|c|c|c|c|c|c|c|}
\hline \multirow{3}{*}{ Atributo } & \multicolumn{6}{|c|}{ Profundidade } \\
\hline & \multicolumn{3}{|c|}{$15 \mathrm{~cm}$} & \multicolumn{3}{|c|}{$30 \mathrm{~cm}$} \\
\hline & Média (\%) & S (\%) & CV (\%) & Média (\%) & S (\%) & CV (\%) \\
\hline PD 1 & 29,70 & 2,46 & 8,29 & 31,86 & 2,40 & 7,56 \\
\hline PC 1 & 28,50 & 2,13 & 7,50 & 31,96 & 2,24 & 7,03 \\
\hline PD 2 & 31,34 & 2,42 & 7,75 & 32,27 & 2,49 & 7,72 \\
\hline PC 2 & 31,34 & 2,03 & 6,50 & 33,13 & 2,52 & 7,62 \\
\hline PD 3 & 28,41 & 2,33 & 8,23 & 28,94 & 2,59 & 8,95 \\
\hline PC 3 & 27,56 & 2,07 & 7,54 & 27,41 & 1,56 & 5,72 \\
\hline PD 4 & 30,12 & 3,25 & 10,82 & 33,91 & 2,85 & 8,41 \\
\hline PC 4 & 29,41 & 2,32 & 7,92 & 34,02 & 2,15 & 6,34 \\
\hline
\end{tabular}

PD1, PD2, PD3, PD4 - umidade do solo no Plantio Direto em 4 épocas de amostragens, respectivamente; PC1, PC2, PC3, PC4 - umidade do solo no Plantio Convencional em 4 épocas de amostragens, respectivamente; S: Desvio padrão e CV: Coeficiente de variação

Verifica-se, a partir do valor do coeficiente de variação, que a umidade do solo apresentou baixa variabilidade, ou seja, existe pequena dispersão dos valores observados em torno da média. Comparando-se os dois sistemas de plantio e as duas profundidades, vê-se que a variabilidade avaliada pelo coeficiente de variação praticamente não diferiu de um sistema para outro nem de uma profundidade para outra, apesar do plantio direto sempre apresentar tendência de maiores CV's; entretanto, para se ter uma avaliação melhor desta dispersão, avaliaram-se os semivariogramas dos tratamentos estudados. Verifica-se ainda que, quando se comparam as profundidades, o coeficiente de variação apresenta maiores valores na profundidade de $15 \mathrm{~cm}$.

Guimarães (1993), no estudo da variabilidade espacial da umidade do solo através da sonda de neutrôns em um Latossolo Roxo na região de Campinas, São Paulo, verificou baixos coeficientes de variação para esse atributo nas profundidades de 30 e $60 \mathrm{~cm}$.

Os dados da umidade do solo foram superiores na profundidade de $30 \mathrm{~cm}$, em ambos os sistemas de manejo, den- tre os quais se observa que, na profundidade de $15 \mathrm{~cm}$, a umidade de água está sensivelmente mais acentuada para o plantio direto. Vieira \& Muzilli (1984) concluíram que a diferença entre o plantio direto e outros sistemas de preparo reside no fato de que a superfície do solo neste sistema é protegida por camada de cobertura morta não revolvida, apresenta estrutura rígida, mais resistente à deformação que a camada superficial em sistema de preparo com revolvimento; além disso, o solo no plantio direto permanece, após uma chuva, com graus mais elevados de umidade por maior período de tempo.

Blevins et al. (1990) constataram, em diferentes condições ambientais, maior disponibilidade de água no plantio direto em relação ao preparo convencional, diferença bastante sensível na camada superficial do solo $(0-20 \mathrm{~cm})$. Canavalle \& Roloff (1997) verificaram menor disponibilidade de água na camada arável sob sistema de plantio direto, em relação à camada de 20 e $40 \mathrm{~cm}$.

Ribeiro Júnior (1995) citou que os valores para a variável umidade do solo estão relacionados com a profundidade mas as variabilidades não acompanham esta tendência. Sidiras et al. (1984) observaram umidade volumétrica semelhante entre os preparos de solo na profundidade de $30 \mathrm{~cm}$, com leve acentuação para o plantio convencional, além de uma retenção maior de água, na camada superficial para o plantio direto.

Apresentam-se, na Tabela 2, os testes de normalidade de Anderson-Darling e os coeficientes de assimetria e curtose para o teste de Jones, a nível de 5\% de significância. $\mathrm{Na}$ análise avaliou-se a hipótese de que a distribuição dos dados é normal.

Tabela 2. Testes de Normalidade para a umidade do solo nas parcelas em plantio direto (PD) e plantio convencional (PC), em quatro coletas de dados e duas profundidades 15 e $30 \mathrm{~cm}$

\begin{tabular}{|c|c|c|c|}
\hline \multirow{2}{*}{$\begin{array}{c}\text { Atributo } \\
\text { Umidade do solo }\end{array}$} & \multirow{2}{*}{$\begin{array}{c}\text { Teste de Normalidade } \\
\text { de Anderson-Darling } \\
\text { (p-valor) }\end{array}$} & \multicolumn{2}{|c|}{$\begin{array}{l}\text { Teste de Jones } \\
\text { (amostral) }\end{array}$} \\
\hline & & Assimetria & Curtose \\
\hline PD 1 - 15 & $0,063^{*}$ & $-0,012^{*}$ & $-0,840^{\star}$ \\
\hline PD 1 - 30 & $0,487^{*}$ & $0,065^{\star}$ & $-0,641^{*}$ \\
\hline PC $1-15$ & $0,085^{*}$ & $0,368^{\star}$ & $-0,723^{\star}$ \\
\hline PC 1 - 30 & 0,011 & $-0,639^{*}$ & $-0,243^{*}$ \\
\hline PD 2 - 15 & $0,283 *$ & $0,169 *$ & $0,007^{*}$ \\
\hline PD 2 - 30 & 0,009 & $-0,180^{*}$ & $-1,186$ \\
\hline PC 2 - 15 & $0,190^{*}$ & $0,246^{*}$ & $-0,477^{\star}$ \\
\hline PC 2 - 30 & 0,341 * & $0,316^{*}$ & $0,150^{*}$ \\
\hline PD 3 - 15 & $0,907^{*}$ & $-0,079^{\star}$ & $-0,497^{*}$ \\
\hline PD 3 - 30 & 0,006 & $-0,570^{*}$ & $-0,151^{*}$ \\
\hline PC $3-15$ & $0,266^{*}$ & $0,165^{\star}$ & $-0,815^{\star}$ \\
\hline PC 3 - 30 & $0,292^{*}$ & $-0,043$ * & $-0,950$ \\
\hline PD 4 - 15 & $0,670^{*}$ & $0,084^{*}$ & $0,386^{*}$ \\
\hline PD 4 - 30 & 0,002 & $-0,579^{*}$ & $-0,740$ * \\
\hline PC $4-15$ & 0,008 & $-0,296^{*}$ & $-1,090$ \\
\hline PC $4-30$ & $0,576^{*}$ & $-0,388^{*}$ & $-0,103^{*}$ \\
\hline
\end{tabular}

*: a variável tem distribuição normal a nível de 5\% de significância. PD1-15, PD2-15, PD3-15, PD4-15: umidade do solo no Plantio Direto em 4 épocas de amostragens na profundidade de $15 \mathrm{~cm}$; PC1-15, PC2-15, PC3-15, PC4-15: umidade do solo no Plantio Convencional em 4 épocas de amostragens na profundidade de $15 \mathrm{~cm}$; PD1-30, PD2-30, PD3-30, PD4-10: umidade do solo no Plantio Direto em 4 épocas de amostragens na profundidade de $30 \mathrm{~cm}$; PC1-30, PC2-30, PC3-30, PC4-30: umidade do solo no Plantio Convencional em 4 épocas de amostragens na profundidade de $15 \mathrm{~cm}$ 
Segundo os testes de normalidade de Anderson-Darling e de Jones, de assimetria e curtose, as variáveis PD2 na profundidade de $30 \mathrm{~cm}$ e PC4 na profundidade de $15 \mathrm{~cm}$, não apresentaram distribuição normal. Para elas, realizou-se a transformação de dados segundo Box \& Cox (1964). As demais variáveis mostraram normalidade em um dos testes, a nível de 5\% de significância; os valores se encontram entre $\pm 0,582$ para assimetria e $-0,84$ e 1,45 para curtose, conforme a tabela de dados amostrais de Jones (1969).

\section{Variabilidade espacial}

Apresentam-se, Tabela 3, os modelos ajustados e seus respectivos parâmetros aos semivariogramas experimentais da umidade do solo em cada sistema, época e profundidade. Os semivariogramas apresentaram três tipos de modelo ajustados: exponencial, esférico e gaussiano. Os modelos mostraram dependência espacial entre moderada e fraca, verificado pelo grau de dependência espacial que se manteve entre 0,25 e 0,75 , observado pelos valores de efeito pepita relativo $\varepsilon=\mathrm{C}_{0} /\left(\mathrm{C}_{1}+\mathrm{C}_{0}\right)$. Os modelos produziram alcances variando entre 5,3 e 10,7 m, ou seja, as amostras são independentes espacialmente, acima desses valores.

Tabela 3. Modelos de semivariogramas ajustados aos dados experimentais da umidade solo, em quatro épocas de amostragem (1, 2, 3 e 4), duas profundidades $(15$ e $30 \mathrm{~cm}$ ) e dois sistemas de plantio (plantio direto e preparo convencional)

\begin{tabular}{ccccccc}
\hline Atributo & $\begin{array}{c}\text { Prof. } \\
\text { (cm) }\end{array}$ & Modelo & $\mathbf{C}_{\mathbf{0}}$ & $\mathbf{a}$ & $\mathbf{C}_{\mathbf{0}}+\mathbf{C}_{\mathbf{1}}$ & $\boldsymbol{\varepsilon}=\mathbf{C}_{\mathbf{0}} /\left(\mathbf{C}_{\mathbf{0}}+\mathbf{C}_{\mathbf{1}}\right)$ \\
\hline PD 1 & 15 & Exp & 4,50 & 6,11 & 5,58 & 80,6 \\
\hline PD 2 & 15 & Exp & 4,11 & 5,20 & 6,02 & 68,2 \\
\hline PD 3 & 15 & Exp & 3,56 & 5,30 & 4,77 & 74,6 \\
\hline PD 4 & 15 & Esf & 4,94 & 9,48 & 11,20 & 44,1 \\
\hline PD 1 & 30 & Esf & 1,91 & 7,48 & 6,38 & 30,0 \\
\hline PD 2 & 30 & Exp & 4,15 & 5,72 & 6,38 & 65,0 \\
\hline PD 3 & 30 & Exp & 5,22 & 6,16 & 7,56 & 69,0 \\
\hline PD 4 & 30 & Esf & 3,80 & 6,49 & 8,99 & 42,3 \\
\hline PC 1 & 15 & Exp & 3,00 & 8,06 & 4,61 & 65,0 \\
\hline PC 2 & 15 & Exp & 2,30 & 8,58 & 4,06 & 56,6 \\
\hline PC 3 & 15 & Esf & 3,01 & 7,02 & 4,60 & 65,4 \\
\hline PC 4 & 15 & Exp & 2,41 & 7,92 & 5,26 & 45,8 \\
\hline PC 1 & 30 & Gau & 2,60 & 10,67 & 5,14 & 50,6 \\
\hline PC 2 & 30 & Esf & 4,09 & 8,03 & 6,29 & 65,0 \\
\hline PC 3 & 30 & Esf & 1,04 & 7,37 & 2,54 & 41,0 \\
\hline PC 4 & 30 & Exp & 2,53 & 7,04 & 4,73 & 53,4 \\
\hline
\end{tabular}

$\mathrm{C}_{0}$ - Efeito pepita; $\mathrm{C}_{0}+\mathrm{C}_{1}$ - Patamar; a - Alcance; Esf - Modelo esférico; Exp - Modelo exponencial; Gau - Modelo gaussiano; PD1, PD2, PD3, PD4 - umidade do solo no Plantio Direto em 4 épocas de amostragens, respectivamente; $\mathrm{PC} 1, \mathrm{PC} 2, \mathrm{PC} 3, \mathrm{PC} 4$ - umidade do solo no Plantio Convencional em 4 épocas de amostragens, respectivamente

Resultados semelhantes foram encontrados por Souza et al. (2001), quando apresentaram dados de umidade do solo com coeficiente de variação abaixo de $10 \%$, fraca dependência espacial e alcance de $8,36 \mathrm{~m}$ para um grid de $30 \times 150 \mathrm{~m}$. No estudo de uma grade de 7 × 9 m com os pontos espaçados $20 \mathrm{~m}$, Vieira et al. (1991) observaram baixa variabilidade espacial para a umidade do solo, explicados pelo baixo coeficiente de variação. Guimarães (1993) estudando umidade do solo em grid de 120 x 160 m em três épocas do ci- clo de "Lab-Lab", observou baixa variabilidade espacial para a variável.

O ajuste dos dados experimentais de umidade do solo na profundidade de $15 \mathrm{~cm}$, no sistema de plantio direto, para as quatro épocas (PD1, PD2, PD3 e PD4) apresentou que o modelo exponencial se ajustou às três primeiras coletas, enquanto o modelo esférico foi ajustado para a $4^{\mathrm{a}}$ época de coleta (Tabela 3 ).

Os ajustes dos modelos teóricos aos semivariogramas da umidade do solo para as quatro épocas no plantio direto na profundidade de $15 \mathrm{~cm}$, apresentaram modelos exponencial e esférico e revelam os alcances de: 6,11 e 5,20 e 5,30 e $9,48 \mathrm{~m}$, respectivamente, significando que acima dessas distâncias as amostras são independentes. Verifica-se, ainda, que os valores do efeito pepita $\left(\mathrm{C}_{0}\right)$ estão muito próximos dos valores do patamar $\left(\mathrm{C}_{0}+\mathrm{C}_{1}\right)$. A relação $\varepsilon=\mathrm{C}_{0} /\left(\mathrm{C}_{0}+\mathrm{C}_{1}\right)$ nos diversos tratamentos indica 76 e 68 e 74 e $44 \%$ o que demonstra grau de dependência espacial moderada. Variando a direção do vetor $\mathrm{h}$ em $0^{\circ}$ (na direção $\mathrm{X}$ ), $90^{\circ}$ (na direção $\mathrm{Y}), 45^{\circ}$ e $135^{\circ}$ (diagonais principais), não foram notadas variações nos semivariogramas experimentais em cada condição do experimento, indicando não existir tendência direcional e, conseqüentemente, o semivariograma é isotropia.

Os ajustes dos modelos teóricos aos semivariogramas correspondentes a umidade do solo para as quatro épocas no plantio convencional na profundidade de $15 \mathrm{~cm}$, apresentaram modelos exponencial e esférico, com alcances de 8,06 e 8,58 e 7,02 e 7,92 m, respectivamente, demonstrando que as amostras distanciadas acima desses valores são independentes espacialmente, ou seja, não existe autocorrelação entre as amostras. Os efeitos pepita $\left(\mathrm{C}_{0}\right)$ com valores de 3,00, 2,30, 3,01 e 2,41 são mais distantes do patamar $\left(\mathrm{C}_{0}+\mathrm{C}_{1}\right)$ que os dados do plantio direto $15 \mathrm{~cm}$, implicando em um grau de dependência espacial moderado $(\varepsilon=65,57,65$ e $46 \%$, respectivamente). A variação no vetor h nas direções de 0,45 , 90 e $135^{\circ}$ apresentou isotropia.

A partir do conjunto de informações fornecidas pelos semivariogramas na profundidade de $15 \mathrm{~cm}$, nos sistemas de plantio direto e preparo convencional, observa-se maior alcance médio na dependência espacial entre as amostras do preparo convencional em relação ao plantio direto além, ainda, de leve acentuação no grau de dependência espacial para o plantio convencional.

De modo geral, a partir dos semivariogramas ressalta-se que a variabilidade espacial do atributo umidade do solo é pouco influenciada pelo manejo do solo, considerando-se o primeiro ano de plantio direto com pouca cobertura morta e o preparo convencional sendo mínimo.

Considerando-se que foi estudada somente umidade do solo com acompanhamento das precipitações nos períodos, entende-se que o estudo da análise do efeito do tempo na variabilidade espacial fica inviabilizada, ou seja, seriam necessários estudos de outras variáveis para se fazer a análise temporal da variável.

A Figura 2 apresenta o comportamento espacial da umidade do solo para o plantio direto (PD) na profundidade de $15 \mathrm{~cm}$; nela, observa-se forte semelhança entre os mapas temáticos de distribuição na área, com valores amostrais 
A

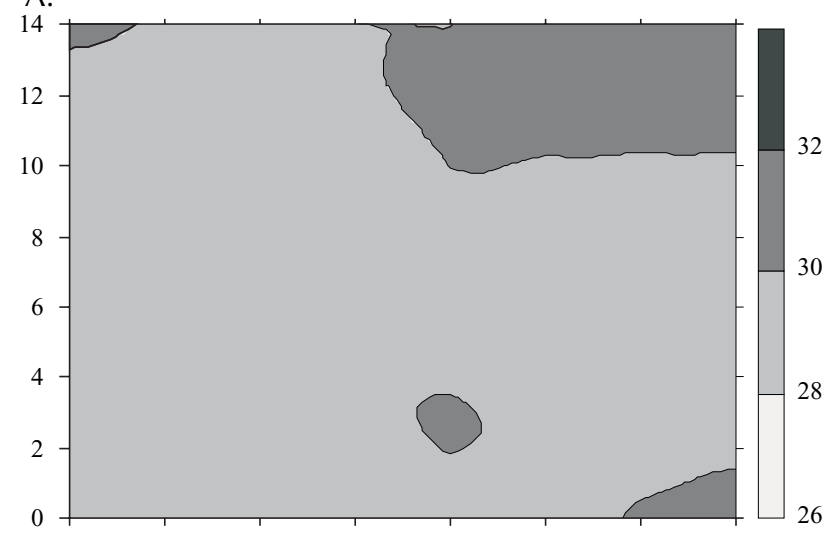

B.

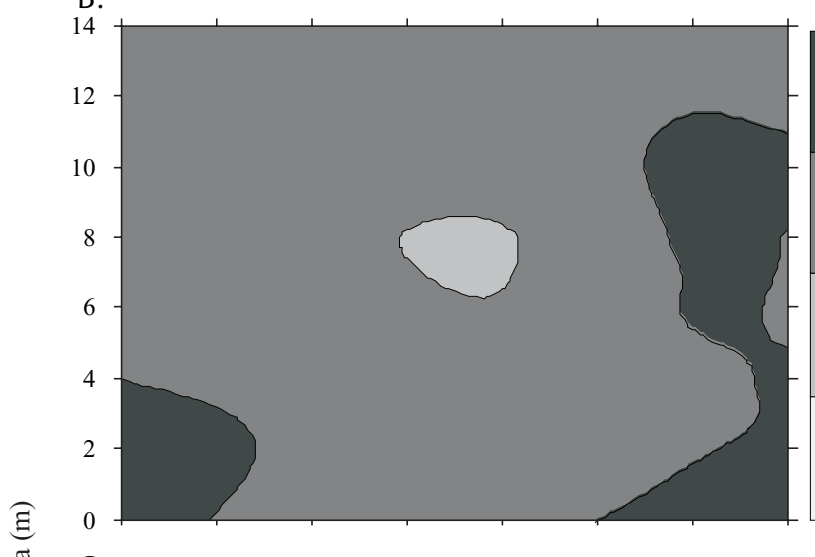

苟

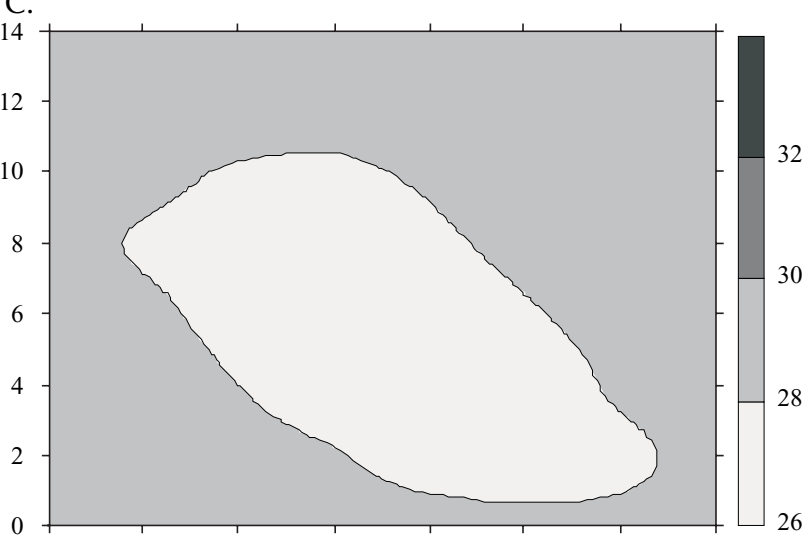

D.

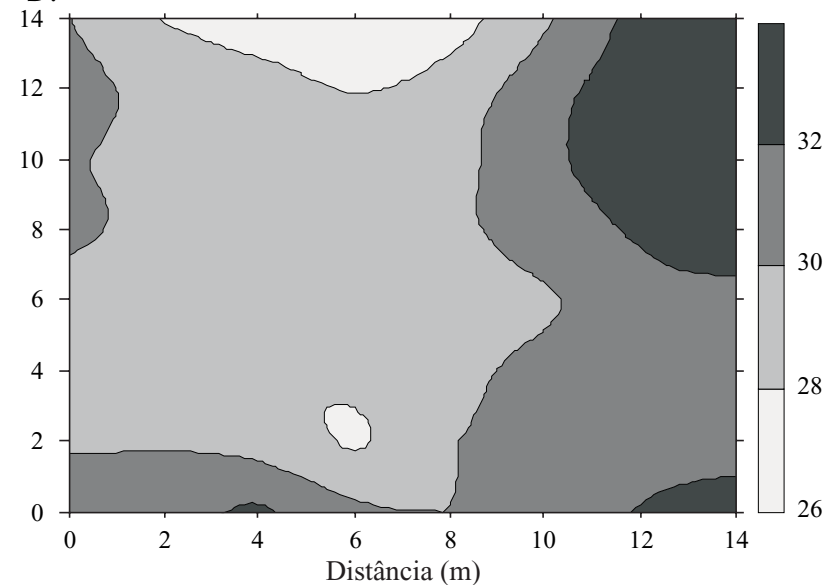

E.

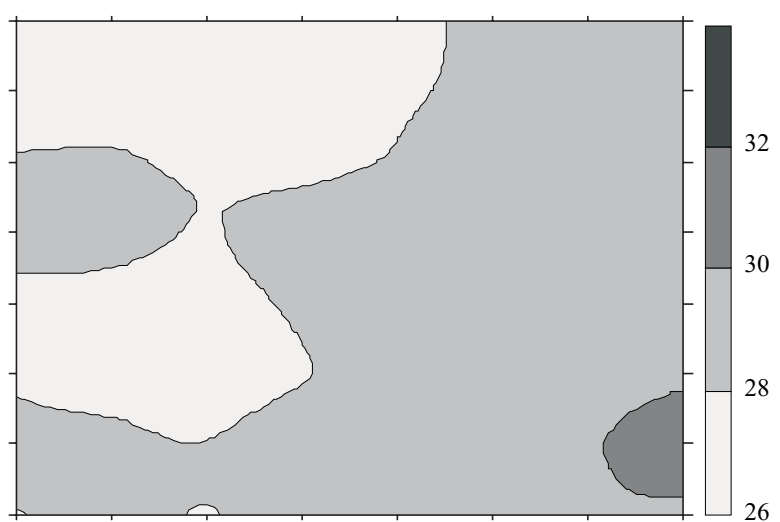

F.

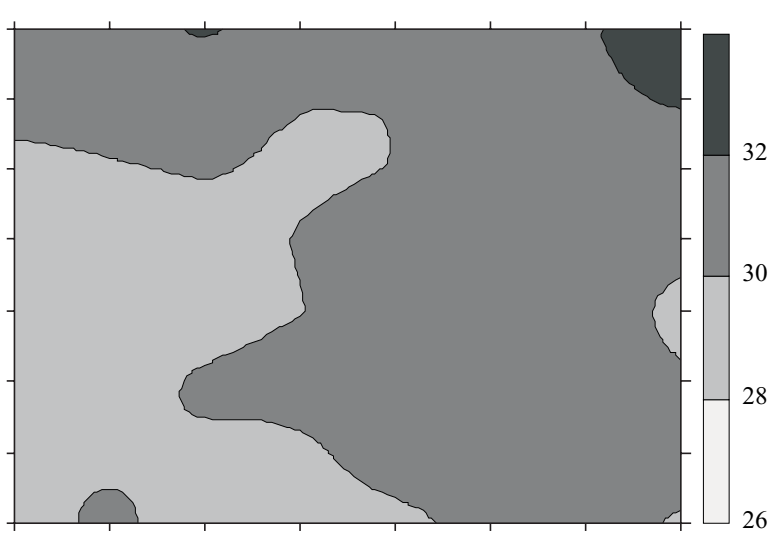

G.

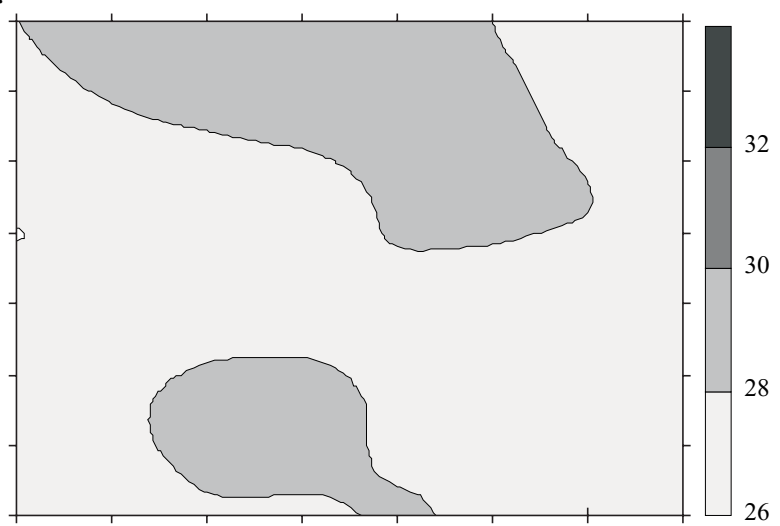

$\mathrm{H}$.

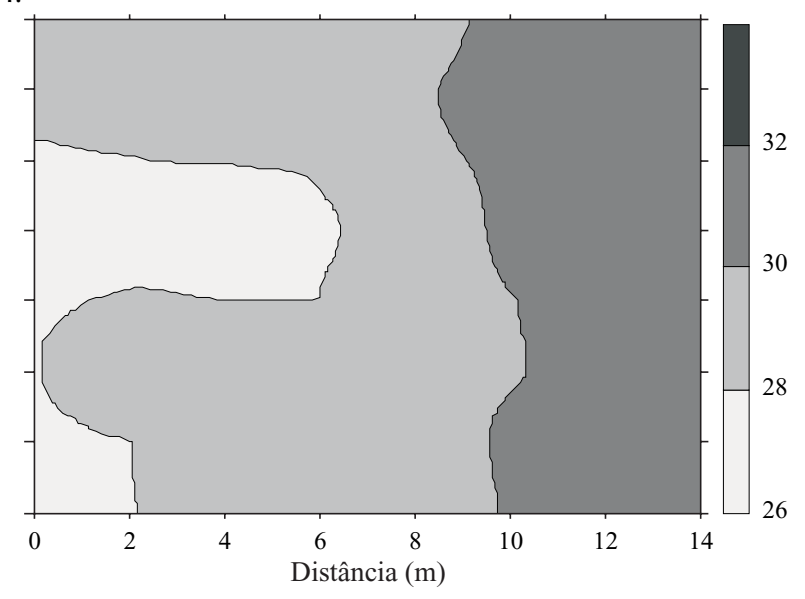

Figura 2. Distribuição espacial da umidade do solo (\%) em quatro épocas de amostragens no sistema de plantio direto (A-PD1; B-PD2; C-PD3; D-PD4) e no sistema de plantio convencional (E-PC1; F-PC2; G-PC3; H-PC4) na profundidade de $15 \mathrm{~cm}$ 


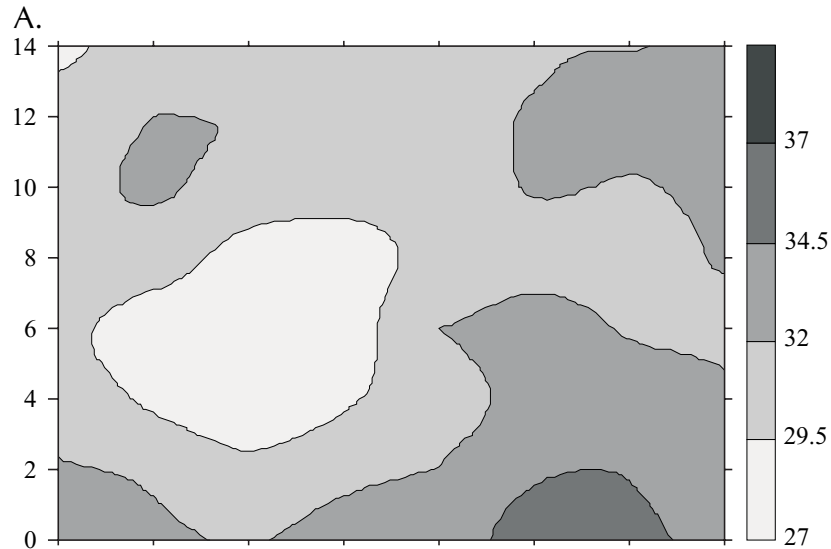

E.
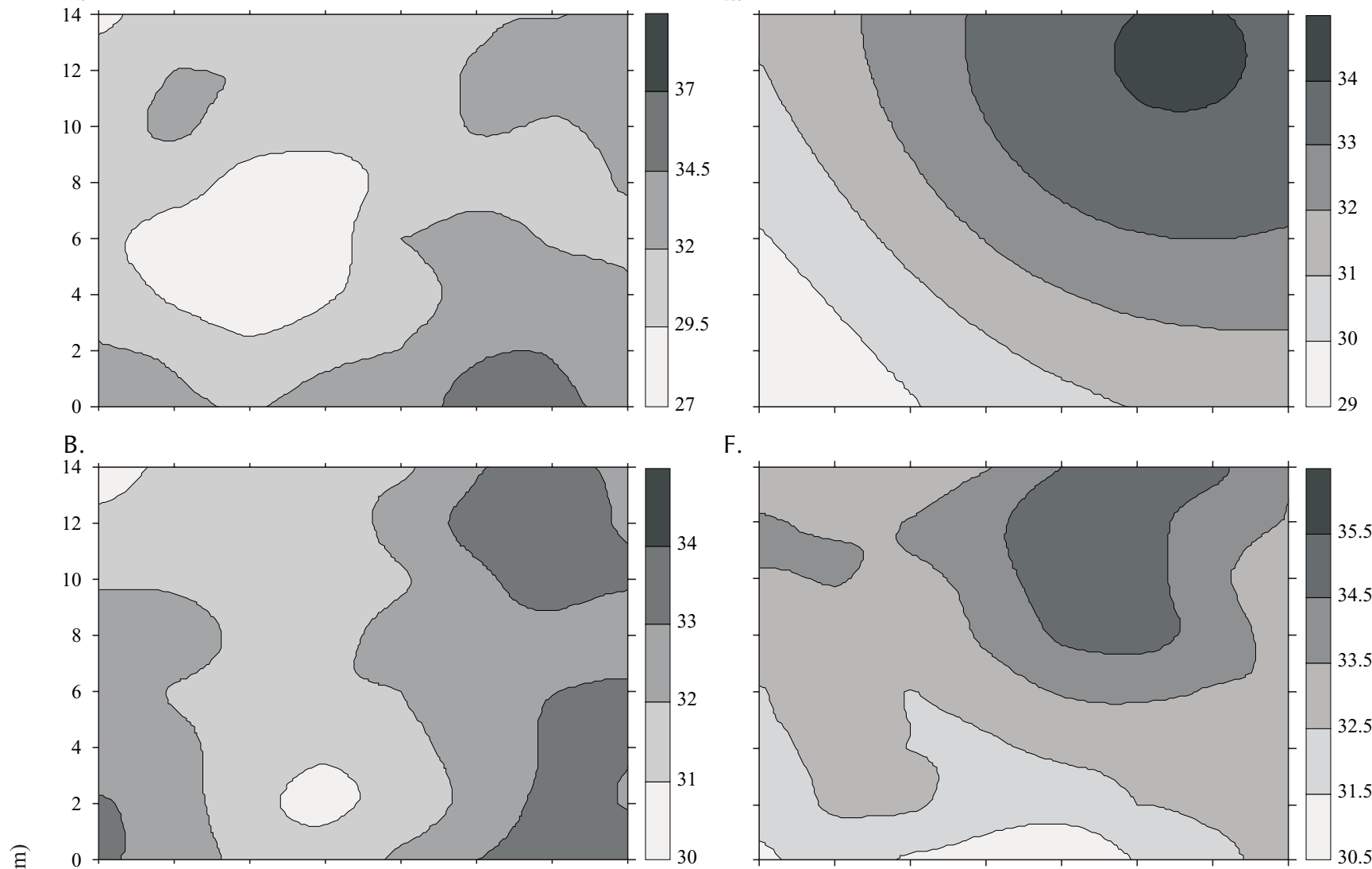

F.
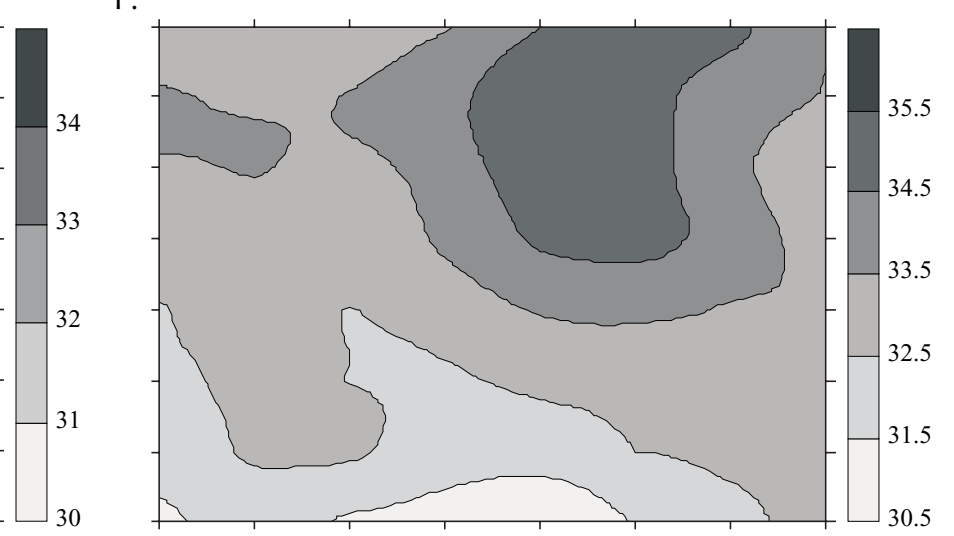

G.
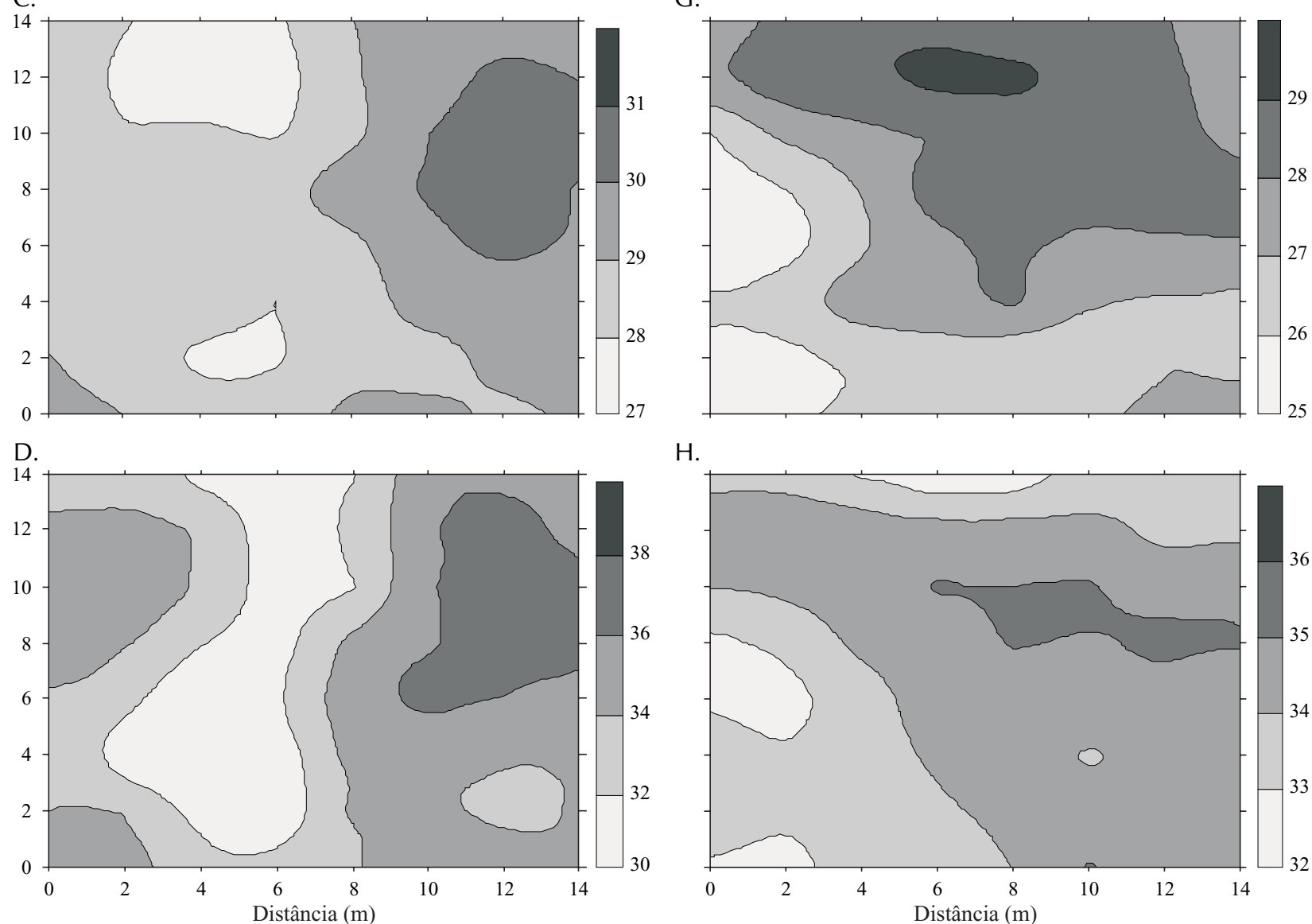

$\mathrm{H}$.

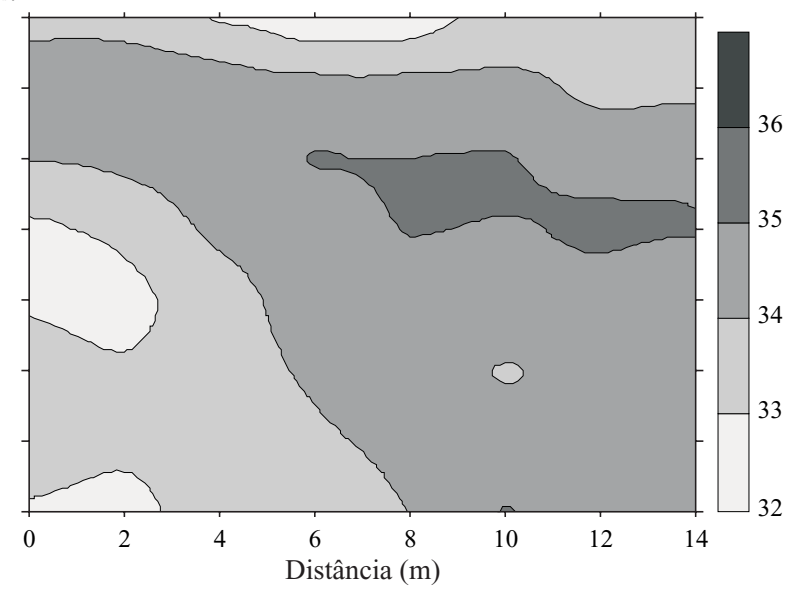

Figura 3. Distribuição espacial da umidade do solo (\%) em quatro épocas de amostragens no sistema de plantio direto (A-PD1; B-PD2; C-PD3; D-PD4) e no sistema de plantio convencional (E-PC1; F-PC2; G-PC3; H-PC4) na profundidade de $30 \mathrm{~cm}$ 
mínimos entre 21 e $26 \%$, valores máximos entre 30 e $37 \%$ e valores entre 26 e $32 \%$. Poucos são os pontos nos valores extremos e a distribuição prevalece entre os valores médios com alguns pontos de maior teor de água no setor direito. Percebem-se amplitudes menores e melhor distribuição na $3^{\mathrm{a}}$ época, o que pode ser justificado pelos baixos índices pluviométricos no período; nota-se, também, na $4^{\text {a }}$ época, maior número de regiões distintas, o que pode ser atribuído aos maiores índices pluviométricos nesta época (Figura 1).

Ainda, na Figura 2 apresentam-se os mapas temáticos do comportamento espacial da umidade do solo para o preparo convencional (PC) na profundidade de $15 \mathrm{~cm}$. Nesta camada e sistema, e se considerando as quatro épocas de amostragens, tem-se os dados amostrais da umidade do solo os quais revelam variação entre 23,35 a $36,43 \%$, e dados ajustados entre 26 e $33 \%$, notando-se progressão na umidade do setor esquerdo para o setor direito, porém não se observam regiões com valores máximos, enquanto a terceira época ou PC3 apresenta distribuição mais homogênea com valores entre 24 e $27 \%$, de modo geral, verifica-se que o comportamento espacial é muito semelhante na área, em todas as épocas de amostragem.

O comportamento espacial da umidade do solo para o plantio direto (PD) na profundidade de $30 \mathrm{~cm}$, é apresentado na Figura 3. Os mapas temáticos se mostram muito heterogêneos entre si, ou seja, não há região predominante com mesma amplitude e apresenta várias regiões com os dados amostrais de amplitudes entre 27, 30 e 33\%. As épocas PD1 e PD3 oferecem um indicativo de melhor distribuição, regiões mais definidas com tendência de amplitude, da esquerda para a direita.

A Figura 3 também mostra o comportamento espacial da umidade do solo para o preparo convencional (PC) na profundidade de $30 \mathrm{~cm}$. Os mapas temáticos apresentam tendência de maior umidade de baixo para cima e da esquerda para a direita; ao longo das épocas de amostragem a distribuição se torna mais homogênea. Verificam-se, pelos dados amostrais, regiões predominantes com amplitudes entre 30 e $33 \%$; em PD3 a amplitude média se apresenta menor.

De maneira geral se constata nos mapas temáticos, que a distribuição espacial da umidade do solo é próxima da média em cada sistema e amostragem, não existindo tendências para um ou outro sistema de manejo, mas não se verificam regiões para valores máximos e mínimos dos dados. A melhor distribuição espacial na $3^{\mathrm{a}}$ época de amostragem foi atribuída à menor precipitação no período, como melhor distribuição na profundidade de $30 \mathrm{~cm}$ em ambos os sistemas, justificado pelo maior grau de dependência espacial encontrado a esta profundidade.

Prevalecem regiões de maior amplitude no setor direito dos mapas temáticos e a investigação revela leve depressão apresentada no terreno nessas coordenadas. Resultados semelhantes foram encontrados por Johann (2001) em variabilidade espacial dos atributos do solo, na região Oeste do Paraná. Em sistemas com e sem manejo químico localizado, verificou-se que o padrão de variabilidade é semelhante quanto à distribuição na área para os dois métodos de manejo com os teores de água variando entre 30 e $32 \%$.
Guimarães (1993) concluiu, em estudo sobre a umidade do solo de um Latossolo Roxo na região de Campinas, SP, que ocorre estabilidade de distribuição da água do solo com o tempo, encontrando amplitudes entre 20 e $30 \%$. Para diferentes condições ambientais, Blevins et al. (1990), notaram maior disponibilidade de água para solos com plantio direto em relação ao plantio convencional, na razão de 36 a 45\%.

\section{CONCLUSÕES}

1. Os valores de umidade estão relacionados à profundidade, mas as variabilidades não acompanham esta tendência.

2. Na camada superficial de $15 \mathrm{~cm}$, o sistema plantio direto apresenta maiores amplitudes da umidade, e o preparo convencional, maior grau na dependência espacial entre os dados amostrados.

3. Na camada mais profunda, de $30 \mathrm{~cm}$, não se observam tendências no grau de umidade nem variabilidade espacial para um dos sistemas de manejo, isto é, o sistema de manejo na profundidade de $30 \mathrm{~cm}$ não influencia na umidade do solo nem na variabilidade espacial.

4. A umidade do solo apresenta grau de dependência espacial entre as amostras, de moderado para fraco, em ambos os sistemas e profundidades.

\section{LITERATURA CITADA}

Blevins, R. L.; Frye, W. W.; Baldwin, P. L.; Robertson, S. D. Tillage effects on sediment and soluble nutrient losses from a Maury silt loam. Journal Environment Quality, Madison, v.19, p.683-686, 1990.

Box, G. E. P.; Cox, D. R. Analysis of transformations (with discussion). Journal of the Royal Statistics Society, Brighton, n.26, p.211-252, 1964.

Canavalle, L. B; Roloff, G. Influência do preparo e da correção do solo na condição hídrica de um Latossolo Vermelho-escuro sob plantio direto. Revista Brasileira de Ciência do Solo, Campinas, v.21, n.1, p.1-6, 1997.

Clark, I. Practical geoestatistics. London: Applied science publishers Ltda, 1979. 123p.

Cressie, N. A. Statistics for spatial data. New York: John Wiley, 1991. 900p.

EMBRAPA - Empresa Brasileira de Pesquisa Agropecuária. Sistema brasileiro de classificação dos solos. Rio de Janeiro: Embrapa-CNPS, 1997. 212p.

EMBRAPA - Empresa Brasileira de Pesquisa Agropecuária. Sistema brasileiro de classificação dos solos. Brasília: EmbrapaCNPS, 2000. 412p.

Golden Software, INC. Surfer for Windons-User guide. Colorado: Golden Software, Inc., 1997. 340p.

Guimarães, E. C. Variabilidade espacial da umidade e da densidade do solo em um Latossolo Roxo. Campinas: UNICAMP, 1993. 135p. Dissertação Mestrado

Issaks, H. E.; Srivastava, R. M. A introduction to applied geostatistics. Oxford: Oxford University Press, 1989. 560p. 
Johann, A. J. Variabilidade espacial dos atributos do solo. Cascavel: UNIOESTE, 2001. 89p. Dissertação Mestrado

Jones, T. A. Skewness and kurtosis as criteria of normality in observed frequency distribuitions. Journal Sedimentary Petrologgy, Northeast Georgia, v.39, p.1622-1627, December, 1969.

Krige, D. G. A statistical approch to some basic mine evalutation problems on the Witwatersrand. Johanesburg Chemistry Metallurgy Mining Society South African, Johanesburg, v.52, n.6, p.119-139, 1951.

Pannatier, Y. Software for epatial data analysis in 2D. New York: Springer-Verlag, 1996. 96p.

Ribeiro Jr., P. J. Métodos geoestatísticos no estudo da variabilidade espacial de parâmetros do solo. Piracicaba: ESALQ, 1995. 99p. Dissertação Mestrado

Sidiras, N; Vieira, S. R; Roth, C. H. Determinação de algumas características físicas de um Latossolo Roxo distrófico sob plantio direto e preparo convencional. Revista Brasileira de Ciência do Solo, Campinas, v.8, p.265-268, 1984.
Souza, E. G.; Johann, J. A.; Rocha, J. V.; Ribeiro, S. R. A.; Silva, M. S.; Opazo, M. A. U.; Molin, J. P.; Oliveira, E. F.; Nóbrega, L. H. P. Análise da variabilidade espacial dos atributos do solo em uma área experimental Parte I: Análise do P, K, MO, e pH. Engenharia Agrícola, Jaboticabal, v.3, p.80-92, 1999.

Souza, M. Z; Silva, M. Z; Guimarães, G. L; Campos, D. T. S.; Carvalho, M. P; Pereira, T.G. Variabilidade espacial de atributos físicos em um Latossolo Vermelho distrófico sob semeadura direta em Selvíria - MS. Revista Brasileira de Ciência do Solo, Viçosa, v.25, n.3, p.699-707, 2001.

Vieira, M. J.; Muzilli, O. Características físicas de um Latossolo Vermelho-Escuro sob diferentes sistemas de manejo. Pesquisa Agropecuária Brasileira, Brasília, v.19, n.7, p.873-882, 1984.

Vieira, S. R. Geoestatística aplicada à agricultura de precisão. In: GIS Brasil, Curitiba. 1998, 53p.

Vieira, S. R; Nascimento, P. C; Sarvasi, F. O. C; Moura, E.G. Umidade e temperatura da camada superficial do solo em função da cobertura morta por resteva de soja em plantio direto. Revista Brasileira de Ciência do Solo, Viçosa, v.15, n.2, p.219-224, 1991. 